\title{
Ambulatory percutaneous nephrolithotomy in Canada: A cost-reducing innovation
}

\author{
Tad Kroczak, MD, FRCSC ${ }^{1}$; Kenneth T Pace, MD, MSc, FRCSC ${ }^{1}$; Sero Andonian, MD, \\ MSc, FRCSC, FACS ${ }^{2}$; Darren Beiko, MD, MBA, FRCSC ${ }^{3}$ \\ ${ }^{1}$ Division of Urology, Department of Surgery, University of Toronto, Toronto, ON; ${ }^{2}$ Division of Urology, \\ Department of Surgery, McGill University, Montreal, QC; ${ }^{3}$ Department of Urology, Queen's University, \\ Kingston, ON; Canada
}

Acknowledgements: This work was supported in part by the Northeastern AUA Young Investigator Award and Montreal General Hospital Foundation Award to Sero Andonian and the Academic Health Sciences Centre (AHSC)-Alternate Funding Payment (AFP) Innovation Fund Research Grant to Darren Beiko.

Cite as: Can Urol Assoc J 2018 September 27; Epub ahead of print. http://dx.doi.org/10.5489/cuaj.5416

Published online September 27, 2018

$* * *$

Globally, growth in healthcare expenditures is becoming unsustainable. In 2017, Canada's healthcare expenditure was estimated at $\$ 242$ billion (\$6,604 per person) or $11.5 \%$ of gross domestic product. ${ }^{1}$ In Canada, provinces spend on average $40 \%$ of their total budgets on healthcare, and hospitals accounted for the largest proportion (28.3\%) of healthcare dollars. ${ }^{1}$ Over the past few decades there has been a major worldwide shift from inpatient to outpatient surgical care across different specialties. ${ }^{2}$ Patient safetyspecifically reducing the incidence of nosocomial infections - and cost containment have been significant drivers of this shift.

Among surgical specialties, urology has been one of the leaders in advancing ambulatory surgical care. Presently, most penoscrotal and endoscopic urologic operations are routinely performed on an outpatient basis. Yet patients undergoing percutaneous nephrolithotomy (PCNL) continue to routinely require hospitalization despite the introduction of tubeless PCNL in $1997^{3}$ and an initial report of outpatient PCNL dating back to $1986 .{ }^{4}$ Over the past decade, ambulatory PCNL (aPCNL) has emerged as a safe and efficacious option for select patients according to previously published strict criteria in small case series. ${ }^{5,6}$ Since these case series, a few cohort studies have been completed, all of which confirm the safety and efficacy of aPCNL. ${ }^{7-9}$ Based on these studies, the recent Medicare Ambulatory Surgical Center Fee Schedule increase in the United States ${ }^{10}$ and the Affordable Care Act's initiative to augment quality of care and patient satisfaction scores, ${ }^{11}$ there has been a growing interest in performing PCNL on a completely outpatient basis in the United States. 
In Canada, the Canadian Institute for Health Information (CIHI) is an independent, not-for-profit organization that provides essential information on Canadian healthcare systems. According to CIHI's most recent 2016-2017 databases and reports, the average daily cost of a standard hospital bed is $\$ 799 .{ }^{12,13}$ This is based on CIHI's calculated average cost of a standard hospital stay in Canada of $\$ 5,992^{12}$ and CIHI's calculated average length of stay (LOS) in hospital of 7.5 days. ${ }^{13} \mathrm{CIHI}$ does not report PCNL-specific data. Instead, PCNL data is captured within CIHI's larger category termed "Major Intervention on Upper Urinary Tract". The average acute LOS for PCNL and other major interventions on the upper urinary tract in adults is 4.19 days according to CIHI. ${ }^{14}$ Based on CIHI's 2016-2017 databases and provincial data, an estimated 1,600 PCNL operations occur in adults annually in Canada. ${ }^{14}$

If we consider a few reasonable assumptions (first, PCNL patients are admitted to a standard [non-intensive care unit] bed postoperatively and second, when comparing standard PCNL and aPCNL, equivalent or similar: anaesthetic equipment and costs; surgical equipment and costs; physician cost/billings; operative times; perioperative imaging tests and costs; postoperative emergency room visits; postoperative readmissions to hospital; postoperative complication rates [and associated costs for all]), calculation of the cost of an average postoperative PCNL hospital stay will provide an estimate of the cost savings of aPCNL . According to CIHI, the overall estimated average cost of the PCNL surgery is $\$ 9,673 .{ }^{14}$ Given an average cost of a standard hospital bed of $\$ 799$ per day and an average LOS for PCNL of 4.19 days, the.resulting average cost of the hospital stay portion of PCNL is calculated to be $\$ 3,348$. When all the costs are considered the per-procedure costs of aPCNL is calculated to be $\$ 6,325$ (the overall PCNL cost of $\$ 9,673$ minus the cost of the hospital stay of $\$ 3,348$ ), a $34.6 \%$ cost reduction. If $10 \%$ of PCNL procedures can be performed on an outpatient basis, total potential annual cost savings to Canadian healthcare systems is just over \$535,000 (1600 cases/yr x 10\% aPCNL rate $\mathrm{x} \$ 3,348$ savings/case).

The initial Canadian experiences with aPCNL demonstrated that aPCNL is safe and effective in highly selected patients. ${ }^{5,6}$ As experience was gained in Canada, more medically complicated patients with larger stone burdens and complex renal anatomy underwent aPCNL and the results were excellent; high stone-free rates, low complication rates, and readmissions rates similar to standard PCNL showed that an ambulatory approach is feasible. ${ }^{7}$ Patient selection and technical precision with near-perfect needle puncture at the calyceal tip is critical to ensure success with this approach. ${ }^{15}$ Even when patient selection and surgical technique are optimal, the patient may still require admission. Sur et al reviewed 60 patients undergoing outpatient (aPCNL) and found that $28 \%$ of patients who were planned to be discharged after the procedure, ended up being admitted overnight due to persistent pain symptoms or "social reasons". ${ }^{16}$ No difference in 30 day overall complication rate and emergency department presentation was shown between both groups. Admitted PCNL patients were more likely to have a larger stone 
burden, multiple punctures, and supracostal puncture for access. Stone free rate, computed tomography confirmed, for the outpatient group was 67\%. Fahmy et al reported their experience involving a larger cohort of outpatient PCNL patients. ${ }^{8}$ Overall, 146 of 162 patients (90.1\%) were suitable for aPCNL and successfully discharged home the same day with an average of 8.97 hours post-PCNL, without any additional adverse outcomes. ${ }^{8}$ Taking it one step further, Abbott et al presented their growing experience with tubeless aPCNL performed on 219 patients in an ambulatory surgical center (ASC) setting, and confirmed the safety and efficacy of aPCNL performed outside the hospital environment. ${ }^{9}$ The progression of PCNL from the hospital to ambulatory surgical centers is an innovative and natural progression for maximum cost savings. Further analysis and studies are required to determine the potential cost savings of aPCNL in ASCs.

In Canada alone, an estimated 1600 PCNLs are performed annually. Understandably, not all patients are suitable for same-day discharge following PCNL, and a shift from standard PCNL with nephrostomy tube drainage to tubeless PCNL is the natural first step in adopting aPCNL. A recent meta analysis of 14 randomized controlled studies showed that tubeless PCNL is associated with a significantly shorter hospital stay, shorter time to return to normal activity, lower postoperative pain scores, less analgesia requirement, and reduced urine leakage when compared to standard PCNL. ${ }^{17}$

The potential cost savings of aPCNL are limited if ED visits, readmission rates, and complications are greater than those for standard PCNL. A retrospective review of 50 patients undergoing aPCNL from two Canadian institutions had a re-admission rate of $4 \%,{ }^{7}$ favourably comparable to standard PCNL. Six patients (12\%) presented to the ED within 7 days. No major complications were identified and 9 (18\%) of patients had low grade (I-II) Clavien complications.

There are several assumptions that our cost analysis depends on when comparing the surgical aspect of aPCNL to standard PCNL. These include: similar operative times between aPCNL and PCNL, no difference in surgical or anesthetic equipment, no difference in perioperative imaging and associated costs and no difference in ER visits, readmission rates or complication rates. Furthermore, one limitation of our cost analysis is the general difference in renal drainage post-PCNL; standard PCNL usually involves placement of a nephrostomy tube despite a slow trend toward tubeless PCNL among endourologists, whereas aPCNL usually involves placement of a ureteral stent. Despite these assumptions and limitation, one can readily comprehend aPCNL's immediate cost savings by avoiding one or more days in hospital. A randomized trial with healthy patients and similar stone burdens would help elucidate and better quantify the precise cost effectiveness of aPCNL in the "real world". Surgical complexity and patient factors can also affect outcomes and complication rates after PCNL. Patient selection is critical to ensure the safety of aPCNL. A report by Bagrodia et al interestingly demonstrated that body mass index had no negative impact on efficacy, complication rate, or cost in patients undergoing PCNL. ${ }^{18}$ 
Ambulatory PCNL is but one example of how a new approach—one that challenges the surgical dogma that PCNL patients must be admitted postoperatively—can contribute to cost containment in healthcare systems across the globe. Shifting PCNL from an inpatient to an outpatient procedure results in a 35\% reduction in costs, which translates to a cost savings of over $\$ 3,000$ per PCNL in Canada. With the ongoing trend toward tubeless PCNL and ambulatory surgical care, along with the established safety and efficacy of aPCNL and these reported cost savings, aPCNL represents a timely advance and a new option for urologists to consider in the surgical management of nephrolithiasis. Endourologists around the world have started performing aPCNL, and we invite others to adopt a fiscally responsible approach and consider aPCNL in highly selected patients. 


\section{References}

1. Canadian Institute for Health Information. https://www.cihi.ca/sites/default/files/document/nhex2017-trendsreport-en.pdf. Accessed March 16, 2018.

2. Davis JE. Ambulatory surgery ... how far can we go? Med Clin North Am. 1993;77(2):365-75.

3. Bellman GC, Davidoff R, Candela J, Gerspach J, Kurtz S, Stout L. Tubeless percutaneous renal surgery. J Urol. 1997;157(5):1578-82.

4. Preminger GM, Clayman RV, Curry T, et al. Outpatient percutaneous nephrostolithotomy. J Urol. 1986;136(2):355-7.

5. Shahrour W, Andonian S. Ambulatory percutaneous nephrolithotomy: initial series. Urology. 2010;76(6):1288-92.

6. Beiko D, Lee L. Outpatient tubeless percutaneous nephrolithotomy: the initial case series. Can Urol Assoc J. 2010 Aug;4(4):E86-90.

7. Beiko D, Elkoushy MA, Kokorovic A, et al. Ambulatory Percutaneous Nephrolithotomy: What Is the Rate of Readmission? J Endourol. 2015; 29:410-4.

8. Fahmy A, Rhashad H, Algebaly O, Sameh W. Can percutaneous nephrolithotomy be performed as an outpatient procedure? Arab J Urol. 2017 Jan 20;15(1):1-6.

9. Abbott JE, Silva MV, Davalos JG. Ambulatory percutaneous nephrolithotomy performed in a free-standing surgery center: Outcomes of the first 219 cases. $\mathrm{J}$ Urol 2018;199:e1206.

10. Centers for Medicare \& Medicaid Services. https://www.cms.gov/Medicare/Medicare-Fee-for-ServicePayment/ASCPayment/index.html. Accessed March 16, 2018.

11. Loehrer AP, Chang DC, Scott JW, Hutter MM, Patel VI, Lee JE, Sommers BD. Association of the Affordable Care Act Medicaid Expansion With Access to and Quality of Care for Surgical Conditions. JAMA Surg. 2018 Jan 24:e175568. doi: 10.1001/jamasurg.2017.5568. [Epub ahead of print]

12. Canadian Institute for Health Information. https://yourhealthsystem.cihi.ca/hsp/inbrief?lang=en\#!/indicators/01 5/cost-of-a-standard-hospital-stay/;mapC1;mapLevel2;/. Accessed July 31, 2018.

13. Canadian Institute for Health Information. https://apps.cihi.ca/mstrapp/asp/Main.aspx?Server=apmstrextprd_i\& project $=$ Quick\%20Stats\&uid=pce_pub_en\&pwd=\&evt $=2048001 \&$ visualizationM ode=0\&documentID $=$ C6F8B4144B03958E3AE3CAB5DD440EA7. Accessed July 31, 2018.

14. Canadian Institute for Health Information. https://www.cihi.ca/en/patient-costestimator. Accessed July 31, 2018.

15. D. Beiko, S. Andonian, Getting started with ambulatory PCNL: a CanMEDS perspective, Can. Urol. Assoc. J. 9 (2015) 223e225.

16. Bechis SK, Han D, Abbott J et al. Outpatient PCNL: the UC San Diego Health Experience. Journal of Endourology Published Online: 8 Mar 2018 https://doi.org/10.1089/end.2018.0056 
17. Xun $\mathrm{Y}^{1}$, Wang $\mathrm{Q}^{1}$, $\mathrm{Hu}^{1}{ }^{1}$, Lu $\mathrm{Y}^{1}$, Zhang $\mathrm{J}^{1}$, Qin $\mathrm{B}^{1}$, Geng $\mathrm{Y}^{2}$, Wang $\mathrm{S}^{3}$. Tubeless versus standard percutaneous nephrolithotomy: an update meta-analysis. BMC Urol. 2017 Nov 13;17(1):102.

18. Bagrodia A, Gupta A, Raman JD, Bensalah K, Pearle MS, Lotan Y. Impact of body mass index on cost and clinical outcomes after percutaneous nephrostolithotomy. Urology. 2008 Oct;72(4):756-60. 